\title{
Medikamentöse Therapiemöglichkeiten bei vestibulären Störungen, Nystagmus und zerebellären Ataxien
}

\section{Pharmacotherapy of Vestibular Disorders, Nystagmus and Cerebellar Disorders}

Autoren

Institute
K. Feil', N. Böttcher ${ }^{1}$, O. Kremmyda ${ }^{1}$, C. Muth², J. Teufel ${ }^{1}$, A. Zwergal' ${ }^{1}$, T. Brandt ${ }^{2}$, M. Strupp ${ }^{1}$

Neurologische Klinik und Deutsches Zentrum für Schwindel- und Gleichgewichtsstörungen, Klinikum der Universität München

2 Deutsches Zentrum für Schwindel- und Gleichgewichtsstörungen, Klinikum der Universität München

\author{
Schlüsselwörter \\ - vestibuläre Erkrankungen \\ - Therapie \\ - Nystagmus \\ - Aminopyridine \\ - zerebelläre Ataxien \\ - Acetyl-DL-Leucin \\ Key words \\ vestibular disorders \\ - therapy \\ - nystagmus \\ - aminopyridines \\ - cerebellar ataxia \\ - acetyl-DL-leucine
}

\section{Zusammenfassung}

$\nabla$

Zur Pharmakotherapie von vestibulären Erkrankungen kommen im Wesentlichen folgende Wirkstoffgruppen zum Einsatz: Antivertiginosa, Antikonvulsiva, Antidepressiva, Antiphlogistika, Anti-Menière-wirksame Substanzen, Migräneprophylaktika, Aminopyridine (Kaliumkanalblocker) und Acetyl-DL-Leucin (eine modifizierte essenzielle Aminosäure). Die Behandlung des Symptoms Schwindel und der begleitenden vegetativen Beschwerden wie Übelkeit, Brechreiz oder Erbrechen sollte zeitlich stets begrenzt werden. Bei einem akuten einseitigen Vestibularisausfall verbessern Kortikosteroide die Erholung der peripher vestibulären Funktion, ohne ausreichende Evidenz für eine allgemeine Empfehlung.

Für die Wirksamkeit von Betahistin (16 mg dreimal täglich oder $48 \mathrm{mg}$ dreimal täglich) bei Morbus Menière gibt es bislang keine ausreichende Evidenz, ggf. sollten hierbei höhere Dosierungen angestrebt werden - insbesondere, da in tierexperimentellen Studien eine Verbesserung der Durchblutung des Innenohrs nachgewiesen wurde. Bei der Vestibularisparoxysmie sind Carbamazepin/ Oxcarbazepin wahrscheinlich wirksam, es fehlen aber noch randomisierte kontrollierte Studien (RCTs) dazu. Bei der vestibulären Migräne gibt es bislang keine RCTs zur Wirksamkeit von Betablockern oder Topiramat, so dass hier aufgrund von klinischen Erfahrungen die Therapie in Analogie zur Migräne ohne Aura empfohlen wird.

Aminopyridine werden für die Behandlung von Patienten mit Downbeat-Nystagmus (2 RCTs) und der episodischen Ataxie Typ 2 (EA2, 1 RCT) empfohlen. Die Wirksamkeit von Aminopyridinen wurde in tierexperimentellen und funktionellen Bildgebungsstudien evaluiert. Acetyl-DLLeucin, eine modifizierte essenzielle Aminosäure, verbessert die klinischen Symptome der zerebellären Ataxie (bisher 3 Beobachtungsstudien). Nach tierexperimentellen Studien beschleunigt

\section{Abstract \\ $\nabla$}

There are currently different groups of drugs for the pharmacotherapy of vertigo, nystagmus and cerebellar disorders: antiemetics; anti-inflammatories, antimenieres, and antimigraineous medications and antidepressants, anticonvulsants, aminopyridines as well as acetyl-DL-leucine. In acute unilateral vestibulopathy, corticosteroids improve the recovery of peripheral vestibular function, but currently there is not sufficient evidence for a general recommendation. There is insufficient evidence to support the view that $16 \mathrm{mg}$ t.i.d. or $48 \mathrm{mg}$ t.i. d. betahistine has an effect in Menière's disease. Therefore, higher dosages are recommended. In animal studies, it was shown that betahistine increases cochlear blood flow. In vestibular paroxysmia, oxcarbazepine was effective (one randomized controlled trial (RCT)). Aminopyridines are recommended for the treatment of downbeat nystagmus (two RCTs) and episodic ataxia type 2 (EA2, one RCT). There has been no RCT on the efficacy of beta-blockers or topiramate but one RCT on flunarizine in vestibular migraine. Based on clinical experience, a treatment analogous to that for migraine without aura can be recommended. AcetylDL-leucine improved cerebellar ataxia (two observational studies); it also accelerated central compensation in an animal model of acute unilateral lesion, but RCTs were negative. There are ongoing RCTs on treatment of vestibular paroxysmia with carbamazepine (VESPA), acute unilateral vestibulopathy with betahistine (BETAVEST), vestibular migraine with metoprolol (PROVEMIG), benign paroxysmal positional vertigo with vitamin D (VitD@BPPV), EA2 with 4-aminopyridine versus acetazolamide (EAT-2-TREAT), and cerebellar ataxias with acetyl-DL-leucine (ALCAT). 
es auch die zentrale Kompensation vestibulärer Störungen; randomisierte klinische Studien dazu waren negativ.

Derzeit werden die folgenden klinischen RCTs zu verschiedenen Erkrankungen durchgeführt: Vestibularisparoxysmie (Carbamazepin, VesPa), akute einseitige Vestibulopathie/Neuritis vestibularis (Betahistin, BETAVEST), vestibuläre Migräne (Metoprolol, PROVEMIG), BPPV (Vitamin D, VitD@BPPV), EA2 (Aminopyridin vs. Acetazolamid, EAT-2-TREAT) und zerebelläre Ataxien (Acetyl-DL-Leucin, ALCAT).

\section{Einleitung}

Diese Übersicht konzentriert sich auf die Pharmakotherapie von peripheren und zentralen vestibulären Störungen, d.h. Behandlungsoptionen bei akuter einseitiger Vestibulopathie, Morbus Menière und Vestibularisparoxysmie sowie der vestibulären Migräne. Sie befasst sich auch mit den Fortschritten in der symptomatischen Behandlung von Kleinhirnstörungen, einschließlich der episodischen Ataxie Typ 2 (EA2) und verschiedenen Nystagmusformen.

Die Therapie umfasst medikamentöse, physio- und psychotherapeutische und selten chirurgische Maßnahmen. Die symptomatische medikamentöse Therapie sollte bei akutem heftigen Schwindel zeitlich eng begrenzt sein. Im Vordergrund steht eine Therapie mittels spezifischer Medikamente, wobei folgende Pharmakagruppen zum Einsatz kommen: 1. Antivertiginosa 2. Antikonvulsiva 3. Antidepressiva 4. Antiphlogistica 5. Antimenière-Medikamente 6. Antimigränosa 7. Aminopyridine sowie 8. Acetyl-DL-Leucin

\section{Symptomatische medikamentöse Therapie (Antihista- minika, Anticholinergika, Benzodiazepine)}

Diese Präparate sollten nur wenige Tage gegeben werden, da sie nach tierexperimentellen Befunden die gewünschte zentrale Kompensation einer akuten Funktionsstörung hemmen oder verlangsamen können und zum Teil auch ein gewisses Abhängigkeitspotenzial besitzen (Übersicht in [1 - 3]). Die negativen Auswirkungen auf die zentrale Kompensation wurden in einer tierexperimentellen Studie zum akuten einseitigen Labyrinthausfall gezeigt: 4-Aminopyridin war gut symptomatisch wirksam, verzögerte aber die zentrale Kompensation.[4] Somit sind diese Medikamente nicht oder nur eingeschränkt für eine Langzeitbehandlung geeignet. Nach Abklingen der vegetativen Begleitsymptomatik (insbesondere Übelkeit/Erbrechen) sollten keine Antivertiginosa oder sedierenden Pharmaka mehr gegeben werden.

Eine medikamentöse symptomatische Therapie von im Rahmen von akuten Schwindelerkrankungen auftretenden vegetativen Begleitsymptomen mit Antivertiginosa und Sedativa ( $\bullet$ Tab. 1) ist nur bei folgenden vier Indikationen durchzuführen: 1. akute periphere oder zentrale vestibuläre Störung (Dauer der Behandlung nur wenige Tage), 2. Prävention von Nausea und Erbrechen beim benignen peripheren paroxysmalen Lagerungsschwindel (BPPV) (nur kurzfristig in der Akutphase), 3. Prävention der Bewegungs-/Reisekrankheit (punktuelle Behandlung), 4. zentraler Lage-/Lagerungsschwindel mit Nausea (kurzfristige Behandlung über Tage).

Es ist zu betonen, dass bislang keine kontrollierten klinischen Studien zu diesen Medikamenten vorliegen, die den geforderten Qualitätskriterien entsprechen.
Spezifische medikamentöse Therapie

Zur Therapie einzelner Schwindel- und Nystagmusformen werden die folgenden Medikamente eingesetzt ( $\odot$ Tab.2):

\section{Periphere vestibuläre Schwindelsyndrome}

Die sechs häufigsten peripheren vestibulären Schwindelsyndrome sind:

- Neuritis vestibularis/akute einseitige Vestibulopathie

- Morbus Menière

- Vestibularisparoxysmie

- Benigner peripherer paroxysmaler Lagerungsschwindel (BPPV)

- Bilaterale Vestibulopathie

- Perilymphfistel

Für die bilaterale Vestibulopathie und die Perilymphfistel gibt es bislang keine medikamentösen Therapieoptionen; erstere kann möglicherweise bei ausgewählten Patienten mit einem „vestibular implant" behandelt werden, letztere in Einzelfällen durch einen operativen Verschluss („canal plugging“ bei der Dehiszenz des anterioren Bogengangs) [5]. Beim BPPV kann eine symptomatische Pharmakotherapie der Übelkeit vor Beginn des Befreiungs-/Repositionsmanövers notwendig sein. Der Effekt einer möglichen Sekundärprophylaxe mit Vitamin D wird derzeit im Rahmen einer geplanten randomisierten klinischen Studie untersucht. Bei der bilateralen Vestibulopathie wird intensives und regelmäßiges Balancetraining zur Besserung der zentralen vestibulären Kompensation empfohlen [6].

Tab. 1 Symptomatische Therapie von Schwindel, Nausea und Erbrechen (modifiziert aus [3]).

\begin{tabular}{|c|c|c|}
\hline Pharmaka & Dosis & Mechanismus \\
\hline \multicolumn{3}{|c|}{ Antihistaminika: Mittel der ersten Wahl } \\
\hline Dimenhydrinat & $\begin{array}{l}\text { Dragees }(50-100 \mathrm{mg} \text {, alle } 4-6 \mathrm{~h} \text {, } \\
\text { Tageshöchstdosis } 400 \mathrm{mg}) \\
\text { Suppositorien }(1-2 \times 150 \mathrm{mg} / \mathrm{d} \text {, } \\
\text { Tageshöchstdosis } 400 \mathrm{mg})\end{array}$ & $\begin{array}{l}\text { H1-Rezeptor- } \\
\text { Antagonist } \\
\text { Antihistiamini- } \\
\text { kum der 1. Ge- } \\
\text { neration }\end{array}$ \\
\hline \multicolumn{3}{|c|}{ Sertrone: Mittel der ersten Wahl } \\
\hline Ondansetron & Filmtabletten $(4-8 \mathrm{mg}$, alle $8 \mathrm{~h})$ & $\begin{array}{l}5-\mathrm{HT}_{3} \text {-Rezeptor- } \\
\text { Antagonist }\end{array}$ \\
\hline \multicolumn{3}{|c|}{ Anticholinergika: Mittel der zweiten Wahl } \\
\hline Scopolamin & Transdermal $(1,0 \mathrm{mg} / 72 \mathrm{~h})$ & $\begin{array}{l}\text { Muskarinanta- } \\
\text { gonist }\end{array}$ \\
\hline \multicolumn{3}{|c|}{ Benzodiazepine: Mittel der dritten Wahl/nur bei sehr starker Übelkeit } \\
\hline Diazepam & $\begin{array}{l}\text { Tabletten (5-10 mg, alle } 4 \text { - } 6 \text { h) } \\
\text { Injektionslösung } 10 \mathrm{mg} \text { i. m. }\end{array}$ & $\begin{array}{l}\mathrm{GABA}_{A}-\text { Rezeptor } \\
\text { Modulator }\end{array}$ \\
\hline Clonazepam & Tabletten $(0,5 \mathrm{mg}$ alle $4-8 \mathrm{~h})$ & $\begin{array}{l}\mathrm{GABA}_{A} \text {-Rezeptor } \\
\text { Modulator }\end{array}$ \\
\hline
\end{tabular}


Tab. 2 Spezifische Therapie von Schwindel und Nystagmus. RCT: randomisierte kontrollierte Studie (modifiziert aus [3]).

\begin{tabular}{|c|c|c|c|}
\hline Pharmaka & Indikation & Beispiel für Substanz und Dosierung & Studienlage \\
\hline \multirow[t]{2}{*}{$\begin{array}{l}\text { Antiepileptika } \\
\text { Carbamazepin } \\
\text { Oxcarbazepin }\end{array}$} & $\begin{array}{l}\text { Vestibularisparoxysmie } \\
\text { paroxysmale Dysarthrophonie und } \\
\text { Ataxie bei Multipler Sklerose } \\
\text { Paroxysmale Hirnstammattacken } \\
\text { Obliquus superior Myokymie }\end{array}$ & $\begin{array}{l}\text { Carbamazepin }(400-800 \mathrm{mg} / \mathrm{d}) \\
\text { Oxcarbazepin }(300-900 \mathrm{mg} / \mathrm{d})\end{array}$ & $\begin{array}{l}\text { Anwendungsbeobachtungen } \\
\text { RCT zu Oxcarbazepin bei Vestibularis- } \\
\text { paroxysmie abgeschlossen (gerade } \\
\text { in Auswertung) } \\
\text { Aktuelle RCT zu Carbamazepin (VesPa) }\end{array}$ \\
\hline & vestibuläre Epilepsie & Antikonvulsiva (verschiedene) & $\begin{array}{l}\text { diverse RCTs zu Wirksamkeit bei fokalen } \\
\text { Anfällen }\end{array}$ \\
\hline $\begin{array}{l}\text { Betablocker } \\
\text { Metoprolol }\end{array}$ & vestibuläre Migräne & $\begin{array}{l}\text { zur Prophylaxe } \\
\text { Metoprolol }(50-200 \mathrm{mg} / \mathrm{d})\end{array}$ & Anwendungsbeobachtungen \\
\hline $\begin{array}{l}\text { Antihistaminika } \\
\text { Betahistin }\end{array}$ & M. Menière & $\begin{array}{l}\text { Betahistin-dihydrochlorid } \\
(3 \times 96 \mathrm{mg} / \mathrm{d})\end{array}$ & Anwendungsbeobachtungen \\
\hline $\begin{array}{l}\text { ototoxische } \\
\text { Antibiotika } \\
\text { Gentamicin }\end{array}$ & $\begin{array}{l}\text { M. Menière } \\
\text { Tumarkinsche Otolithenkrisen } \\
\text { („vestibuläre drop attacks“) }\end{array}$ & $\begin{array}{l}\text { Gentamicin ( } 10-40 \mathrm{mg} \text { transtympanal in } \\
\text { Abständen von mindestens } 8-12 \text { Wochen) }\end{array}$ & 2 RCTs \\
\hline \multirow[t]{2}{*}{$\begin{array}{l}\text { Kortikosteroide } \\
\text { Methylprednisolon }\end{array}$} & akute Neuritis vestibularis & $\begin{array}{l}\text { Methylprednisolon } \\
\text { ( } 100 \mathrm{mg} / \mathrm{d} \text {, Dosis jeden } 4 . \text { Tag um } 20 \mathrm{mg} \\
\text { reduzieren) }\end{array}$ & $1 \mathrm{RCT}$ \\
\hline & $\begin{array}{l}\text { akutes Cogan-Syndrom und andere } \\
\text { autoimmunologische Innenohrer- } \\
\text { krankungen }\end{array}$ & $\begin{array}{l}\text { Methyprednisolon } \\
\text { (1000 mg/d i.v., Reduzierung entspre- } \\
\text { chend Verlauf) }\end{array}$ & Anwendungsbeobachtung \\
\hline $\begin{array}{l}\text { Kaliumkanal- } \\
\text { blocker } \\
\text { 4-Aminopyridin }\end{array}$ & Downbeat-Nystagmus & $\begin{array}{l}\text { 4-Aminopyridin }(2-3 \times 5 \mathrm{mg} / \mathrm{d}) \\
\text { 4-Aminopyridin retard/Fampiridin } \\
(1-2 \times 10 \mathrm{mg} / \mathrm{d}) \\
\text { 3,4-Diaminopyridin }(2-3 \times 10 \mathrm{mg} / \mathrm{d})\end{array}$ & 2 RCTs \\
\hline \multirow[t]{3}{*}{ 3,4-Diaminopyridin } & Upbeat-Nystagmus & & Anwendungsbeobachtung \\
\hline & Gangstörung bei zerebellärer Ataxie & $\begin{array}{l}\text { 4-Aminopyridin }(2-3 \times 5 \mathrm{mg} / \mathrm{d}) \\
\text { 4-Aminopyridin retard/Fampiridin } \\
(1-2 \times 10 \mathrm{mg} / \mathrm{d})\end{array}$ & $\begin{array}{l}\text { Anwendungsbeobachtung } \\
\text { Aktuelle RCT (FACEG) }\end{array}$ \\
\hline & Episodische Ataxie Typ 2 & 3,4-Diaminopyridin $(2-3 \times 10 \mathrm{mg} / \mathrm{d})$ & \\
\hline $\begin{array}{l}\text { Carboanhydrase- } \\
\text { hemmer }\end{array}$ & Episodische Ataxie Typ 2 & Azetazolamid $(125-1000 \mathrm{mg} / \mathrm{d})$ & Aktuelle RCT (EAT-2-TREAT) \\
\hline $\begin{array}{l}\text { Kaliumkanal- } \\
\text { aktivator } \\
\text { Chlorzoxazone }\end{array}$ & Downbeat-Nystagmus & Chlorzoxazone $(3 \times 500-750 \mathrm{mg} / \mathrm{d})$ & Anwendungsbeobachtung \\
\hline $\begin{array}{l}\text { Aminosäuren } \\
\text { Acetyl-DL-Leucin }\end{array}$ & Zerebelläre Ataxien & Acetyl-DL-Leucin $(3-5 \mathrm{~g} / \mathrm{d})$ & $\begin{array}{l}\text { Anwendungsbeobachtungen } \\
\text { RCT (ALCAT) (Rekrutierung startet im } \\
\text { Oktober 2015) }\end{array}$ \\
\hline SSRI und andere & phobischer Schwankschwindel & Citalopram $(10-20 \mathrm{mg} / \mathrm{d})$ & Anwendungsbeobachtungen \\
\hline Antidepressiva & $\begin{array}{l}\text { sekundäre psychiatrische Störun- } \\
\text { gen, z. B. Panikattacken oder De- } \\
\text { pression bei primär organischen } \\
\text { Schwindelsyndromen }\end{array}$ & Amitriptylin $(10-20 \mathrm{mg} / \mathrm{d})$ & \\
\hline Baclofen & $\begin{array}{l}\text { Upbeat-Nystagmus und andere } \\
\text { zentrale Nystagmusformen }\end{array}$ & Baclofen (15 - $30 \mathrm{mg} / \mathrm{d})$ & Anwendungsbeobachtungen \\
\hline
\end{tabular}

Neuritis vestibularis/akute einseitige Vestibulopathie

Ursache ist der akute, einseitige Ausfall des N. vestibularis, vermutlich hauptsächlich bedingt durch eine Druckschädigung des Nervs innerhalb seines knöchernen Kanals. Eine virale Genese der Neuritis vestibularis ist wahrscheinlich, konnte aber bislang nicht sicher bewiesen werden [7-11]. Autoptische Studien, die entzündliche Degenerationen des Vestibularisnervs zeigten [12], der Nachweis von Herpes-simplex-Virus-Typ-1-DNA und des „latency-associated transcript“ in vestibulären Ganglienzellen [13 16] sprechen aber für diese Genese.

Die klinische Leitsymptomatik einer akuten einseitigen Vestibulopathie ist ein akut oder subakut („stotternder Beginn“) einsetzender Drehschwindel mit Übelkeit und Erbrechen sowie einer Gangunsicherheit mit Fallneigung zur betroffenen Seite. Dieses Syndrom wurde in der Vergangenheit als Neuritis vestibularis bezeichnet. Da eine Entzündung als wahrscheinliche Ursache der Symptomatik in vielen Fällen jedoch nicht nachgewiesen werden konnte, ist die neue rein deskriptive Definition eine „akute einseitige Vestibulopathie“, basierend auf den Empfehlungen des internationalen Klassifikationsausschusses der internationalen Bárány Society [17].

Wie oben dargestellt, sollte bei einer akuten einseitigen Vestibulopathie eine symptomatische Therapie der vegetativen Begleitsymptomatik über ein bis zwei Tage, in Einzelfällen für drei bis acht Tage verabreicht werden. In einem Tiermodell der akuten einseitigen Vestibulopathie wurde gezeigt, dass der Kaliumkanalblocker 4-Aminopyridin 30 Minuten nach Einnahme in der Akutphase die vestibuläre Imbalance reduziert, auf der anderen Seite verzögert die Gabe jedoch die zentrale Kompensation, weshalb diese nicht empfohlen werden kann [4].

In einer randomisierten klinischen Studie zeigte sich eine Verbesserung der peripheren vestibulären Funktion nach einer akuten einseitigen Vestibulopathie durch eine Monotherapie mit Steroiden (Methylprednisolon 100 mg täglich) [18]. Diese Ergeb- 
nisse werden sowohl durch eine Metaanalyse [19] als auch eine Beobachtungsstudie [20] unterstützt. Eine kürzlich erschienene einfach-verblindete Studie, die Kortikosteroide mit Physiotherapie in der Akutphase einer akuten einseitigen Vestibulopathie verglich, zeigte einen ebenbürtigen Effekt beider Therapien sowohl in Bezug auf den klinischen Verlauf als auch bezüglich der kalorischen vestibulären Funktion und der Wiederherstellung der Otolithenfunktion [21].

Eine frühzeitige Therapie mit Kortikosteroiden scheint den frühen klinischen Verlauf zu verbessern, ohne einen Effekt auf die langfristige Prognose der Erkrankung zu haben. Übereinstimmend dazu geben eine Cochrane-Analyse [22] und eine Metaanalyse [19] keine generelle Empfehlung für den Einsatz von Kortikosteroiden. Diese können offensichtlich nur den kalorischen Verlauf der Erkrankung und die Erholung der vestibulären Funktion beeinflussen [19]; die Effekte auf die Lebensqualität der Patienten wurden bislang noch nicht ausreichend untersucht, sodass weitere klinische Studien notwendig sind [23].

In der klinischen Praxis kann eine dreiwöchige Behandlung mit Kortikosteroiden erwogen werden (z.B. Methylprednisolon $100 \mathrm{mg} / \mathrm{d}$ innerhalb von drei Tagen nach Symptombeginn, Dosisreduktion um $20 \mathrm{mg}$ jeden 4 . Tag) [18].

Derzeit gibt es nur sehr wenige tierexperimentelle Studien zu den Auswirkungen von Pharmaka auf die zentrale vestibuläre Kompensation: Alkohol, Phenobarbital, Chlorpromazin, Diazepam und ACTH-Antagonisten führen zu einer Verzögerung der zentralen vestibulären Kompensation, während Koffein, Amphetamine, Glukokortikoide, Acetyl-DL-Leucin und Betahistin die Kompensation beschleunigten (Übersicht siehe [24]). Betahistin und Acetyl-DL-Leucin kommen als Substanzen zur Verbesserung der vestibulären Kompensation in Betracht [25]. Der Nachweis der Wirksamkeit und des Wirkmechanismus im Rahmen von klinischen Studien ist bislang noch ausgeblieben (drei randomisierte klinische Studien waren negativ). Zusammenfassend ist es bislang noch nicht ausreichend untersucht worden, ob diese Substanzen die zentrale vestibuläre Kompensation beeinflussen und wenn ja, in welchem Ausmaß.

Aktuell gibt es eine laufende klinische Studie zur Wirksamkeit von Betahistin auf die zentrale vestibuläre Kompensation in der akuten einseitigen Vestibulopathie (Betahistin-dihydrochlorid $48 \mathrm{mg}$ dreimal täglich im Vergleich zu Placebo; BETAVEST-Studie).

Bei Patienten mit Neuritis vestibularis konnte ein klinisch relevanter Erfolg einer intensiven Physiotherapie für die vestibulospinale Haltungsregulation in einer prospektiven, randomisierten, kontrollierten Studie gezeigt werden [26]. Dies ist ein wichtiges Behandlungsprinzip zur Verbesserung der zentralen vestibulären Kompensation, das auch durch eine Cochrane-Analyse gestützt wird [6]. Vestibuläre Trainingsprogramme umfassen $[6,26,27]$ :

- willkürliche Augenbewegungen und Fixationen zur Verbesserung der gestörten Blickstabilisation,

- aktive Kopfbewegungen zum Rekalibrieren des vestibulookulären Reflexes und

- Balance-, Zielbewegungen und Gehübungen zur Verbesserung der vestibulo-spinalen Haltungsregulation und Zielmotorik.

Morbus Menière

Ätiologie und Pathophysiologie des Morbus Menière sind trotz vieler Untersuchungen noch nicht abschließend geklärt [2830]. Der pathognomonische histopathologische Befund ist ein Endolymphhydrops [31]. Dieser entsteht infolge einer Imbalance zwischen Produktion und Resorption der Endolymphe. Der erhöhte endolymphatische Druck führt zu einer Ruptur der Endolymphmembran und/oder Öffnung spannungssensitiver nicht selektiver Kationenkanäle [32]. Die infolgedessen erhöhte Kaliumkonzentration im Perilymphraum führt zunächst zur Exzitation und dann Depolarisation.

Klinisch ist der Morbus Menière gekennzeichnet durch rezidivierende, mind. 20 Minuten anhaltende Attacken von Schwindel und jeweils einseitige Hörminderung im Tieftonbereich sowie fluktuierenden Tinnitus und Ohrdruckgefühl. In etwa einem Drittel der Fälle gehen eine Verstärkung des Ohrgeräuschs, des Ohrdruckes und eine Hörminderung dem abrupt einsetzenden Schwindel voraus. Im Verlauf der Erkrankung entwickeln sich meist eine bleibende Hörminderung und ein vestibuläres Defizit auf der betroffenen Seite.

Die Therapie des Morbus Menière zielt auf die Verhinderung der Schwindelattacken. Die Therapieempfehlungen reichen von salzfreier Kost über Diuretika, transtympanale Gentamycinoder Steroidgabe oder Betahistin bis zu verschiedenen operativen Verfahren [28]. Systematische Literaturübersichten haben gezeigt, dass weder salzfreie Diät noch Diuretika [33] einen Therapieeffekt haben. Die Sakkotomie ist nicht wirksam, wie eine Cochrane-Analyse nachwies [34]. Damit sind heute diese drei Therapieverfahren ebenso wie die früher weit verbreitete selektive Neurektomie obsolet.

Folgende Behandlungsmöglichkeiten sind bei Patienten mit Morbus Menière zu empfehlen: Betahistin auf eingeschränkter Datenbasis, transtympanales Gentamicin bei schwerem Verlauf und transtympanale Steroide.

Betahistin: Betahistin ist ein starker H3-Antagonist und ein schwacher H1-Agonist [35, 36]. Man vermutet drei Wirkprinzipien: 1. Der cochleäre Blutfluss erhöht sich vor allem über die H3-Rezeptoren [37]. Betahistin hat pharmakologisch einen hohen First-Pass-Effekt und wird in der Leber zu drei Metaboliten verstoffwechselt. Dabei ist es wichtig zu beachten, dass nicht nur Betahistin, sondern auch seine Hauptmetaboliten Einfluss auf die cochleäre Durchblutung haben [38]. 2. Betahistin erhöht den Histaminumsatz im N. vestibulocochlearis und im vestibulären System über die H3-Rezeptoren. 3. Betahistin verringert den vestibulären Input im peripher-vestibulären System.

Inwieweit diese Effekte sich auf die Erkrankung und die prophylaktische Behandlung der Menière-Krankheit auswirken, ist bislang noch nicht bekannt. Diskutiert werden eine Verbesserung der cochleären Mikrozirkulation und damit eine Normalisierung der Imbalance zwischen Produktion und Resorption der Endolymphe.

Man muss jedoch in Bezug auf derzeit laufende klinische Studien darauf hinweisen, dass der Effekt von Betahistin beim Morbus Menière bislang in keiner randomisierten klinischen Studie, die gültigen Qualitätskriterien entspricht, nachgewiesen wurde. Die BEMED-Studie, eine Dosisfindungsstudie (Placebo im Vergleich zu $16 \mathrm{mg}$ dreimal täglich vs. $48 \mathrm{mg}$ dreimal täglich, Behandlungsdauer 9 Monate) mit 221 Patienten ist zur Publikation eingereicht. Eine Beobachtungsstudie mit 112 Patienten (mittlere Anzahl von Attacken 8/Monat) hat einen besseren Effekt von Betahistin $48 \mathrm{mg}$ dreimal täglich im Vergleich zu Betahistin 16 bis $24 \mathrm{mg}$ jeweils dreimal täglich im Hinblick auf die Attackenfrequenz und -intensität gezeigt [39]. In einer weiteren Beobachtungsstudie zeigten sich sogar noch bessere Effekte bei höherer Dosierung (144 bis $480 \mathrm{mg} / \mathrm{d}$ ) [40]. All diese Studien waren jedoch weder Placebo-kontrolliert noch randomisiert. Die Ergebnisse von Metaanalysen sind ernüchternd, beruhen jedoch in den meisten Fällen auf nicht randomisierten, offenen Studien [41, 42]. 
Ausgehend von den persönlichen Erfahrungen der Autoren und mit dem Wissen einer eingeschränkten Beurteilbarkeit, können in Einzelfällen auch weiter höhere Dosierungen von bis zu $96 \mathrm{mg}$ Betahistin dreimal täglich erwogen werden; bei Nichtansprechen können auch noch höhere Dosierung gegeben werden [40]. Ziel der Therapie ist eine mindestens sechsmonatige Attackenfreiheit. Danach kann die Dosis wieder langsam auf eine Erhaltungsdosis reduziert werden.

Transtympanales Gentamicin: In zwei prospektiven randomisierten klinischen Studien wurde gezeigt, dass die transtympanale Gentamicin-Injektion die Attackenfrequenz reduziert [43, 44]. Diese Ergebnisse wurden durch eine Cochrane-Analyse unterstützt [45]. Allerdings fehlen auch hier Studien [46]. Die Hauptnebenwirkung der Aminoglykoside ist eine audiologische Beeinträchtigung in etwa $20 \%$ der Patienten [47]. Einschränkend ist zu berücksichtigen, dass generell etwa $30 \%$ der Patienten eine bilaterale Beteiligung im Rahmen des Morbus Menière in einem Zeitraum von 5 Jahren entwickeln, was ebenfalls eine Limitation dieser Behandlung darstellt [48].

Transtympanale Steroide: Laut einer Cochrane-Analyse [49] wurde bislang nur eine valide Studie zur Wirksamkeit von transtympanalen Steroiden durchgeführt, die einen Vorteil dieser Behandlung dokumentierte [50]. In einer anderen Studie wurden die Wirkungen von Dexamethason mit einer transtympanalen Gentamicin-Therapie verglichen: Letztere reduzierte Schwindelattacken um 93\%, Dexamethason um 61\% [51].

\section{Vestibularisparoxysmie}

Als Ursache der Vestibularisparoxysmie wird eine hirnstammnahe Gefäßkompression des achten Hirnnervs (N. vestibulo-cochlearis) angenommen [52 -55]. Nachweisen lässt sich dies durch eine MRT-Untersuchung - der Gefäß-Nerv-Kontakt ist jedoch nicht spezifisch, da er bei etwa $30 \%$ gesunder Probanden ebenfalls nachweisbar ist.

Die revidierten Diagnosekriterien für eine Vestibularisparoxysmie sind wie folgt: mindestens fünf spontan auftretende Dreh- und/ oder Schwankschwindelattacken mit einer Dauer bis zu 30 Sekunden (selten länger, selten von bestimmten Kopfbewegungen ausgelöst) mit oder ohne begleitende Ohrsymptomatik (Tinnitus und/oder Hörminderung) sowie nicht besser durch eine andere vestibuläre Ursache zu erklären. Bei manchen Patienten lassen sich die Attacken durch Hyperventilation provozieren [52, 53].
Analog zu anderen Erkrankungen, die durch eine neurovaskuläre Kompression verursacht werden, sind Natriumkanalblocker wie Carbamazepin oder Oxcarbazepin theoretisch wirksam. In Beobachtungsstudien wurde gezeigt, dass Therapieversuche mit Carbamazepin in niedriger Dosis $(200-600 \mathrm{mg} / \mathrm{d}$ ) oder Oxcarbazepin $(300-900 \mathrm{mg} / \mathrm{d})$ wirksam und zudem diagnostisch verwertbar sind $[53,56]$. In einer Verlaufsstudie von 32 Patienten über einen mittleren Zeitraum von drei Jahren zeigten sich eine signifikante anhaltende Reduktion der Attackenfrequenz auf $10 \%$ der Ausgangswerte sowie eine Verminderung der Attackenintensität und -dauer [53]. Eine randomisierte klinische Studie mit Oxcarbazepin zeigte (Daten werden derzeit noch analysiert), dass die Schwindeltage im Vergleich zu Placebo reduziert werden, die Drop-out-Rate betrug jedoch $>50 \%$. Bei Unverträglichkeit stehen als Alternativen Phenytoin oder Valproinsäure zur Verfügung, wobei dazu keine Studiendaten vorliegen. Momentan wird eine multizentrische Placebo-kontrollierte Studie mit Carbamazepin ( $400 \mathrm{mg} / \mathrm{d}$ ) durchgeführt (VesPa) ( $\bullet$ Tab. 3).

Die operative mikrovaskuläre Dekompression kann in Einzelfällen erfolgreich sein [54]. Die Indikation zu dieser operativen Therapie sollte trotzdem sehr zurückhaltend gestellt werden. Einerseits besteht wegen eines intra- oder postoperativen Vasospasmus die Gefahr eines Hirnstamm- oder Innenohrinfarkts (ca. 3-5\%), andererseits kann die betroffene Seite häufig nicht ausreichend sicher bestimmt werden.

\section{Zentrale vestibuläre Schwindelsyndrome}

Zentral vestibulärer Schwindel tritt häufig bei ischämisch bedingten Hirnstamm- oder Kleinhirnschädigungen (akuter Drehoder Schwankschwindel), bei der vestibulären Migräne (rezidivierende Schwindelattacken) und bei Kleinhirnerkrankungen auf, z. B. bei der Episodischen Ataxie Typ 2 (rezidivierende Attacken) oder zerebellären Ataxien (meist progredienter Schwankschwindel mit u. a. Gang- und Standataxie). In den letzten Jahren wurden beträchtliche Fortschritte bei der Behandlung insbesondere von Kleinhirnerkrankungen [57] aufgrund der Verwendung von Aminopyridinen und in jüngerer Zeit der Verwendung der modifizierten essenziellen Aminosäure Acetyl-DL-Leucin erzielt.

Vestibuläre Migräne

Die vestibuläre Migräne ist die häufigste Ursache spontan auftretender Schwindelattacken. Die Lebenszeitprävalenz liegt bei

\begin{tabular}{|c|c|c|c|c|}
\hline \multicolumn{5}{|c|}{ Periphere vestibuläre Störungen } \\
\hline 1 & $\begin{array}{l}\text { Benigner peripherer pa- } \\
\text { roxysmaler Lagerungs- } \\
\text { schwindel }\end{array}$ & VitD@BPPV & Vitamin D vs. Placebo & $\begin{array}{l}\text { Beginn der Patientenrekrutierung: } \\
\text { Herbst } 2015\end{array}$ \\
\hline 2 & Vestibularisparoxysmie & VesPa & $\begin{array}{l}\text { Carbamazepin } \\
\text { ( } 400 \text { mg/d vs. Placebo) }\end{array}$ & $\begin{array}{l}\text { Beginn der Patientenrekrutierung: } \\
\text { Herbst } 2015\end{array}$ \\
\hline 3 & Morbus Menière & BEMED & $\begin{array}{l}\text { Betahistin ( } 48 \text { mg/d vs. } \\
144 \text { mg/d vs. Placebo) }\end{array}$ & $\begin{array}{l}\text { Datenauswertung } \\
\text { (last patient out 23.11.2013) }\end{array}$ \\
\hline 4 & $\begin{array}{l}\text { Akute einseitige Vestibu- } \\
\text { lopathie }\end{array}$ & BETAVEST & $\begin{array}{l}\text { Betahistin }(144 \mathrm{mg} / \mathrm{d} \\
\text { vs. Placebo) }\end{array}$ & Laufende Studie \\
\hline \multicolumn{5}{|c|}{ Zentrale vestibuläre Störungen } \\
\hline 5 & Vestibuläre Migräne & PROVEMIG & $\begin{array}{l}\text { Metoprolol ( } 95 \text { mg/d } \\
\text { vs. Placebo) }\end{array}$ & Laufende Studie \\
\hline 6 & Episodische Ataxie Typ 2 & $\begin{array}{l}\text { EAT-2- } \\
\text { TREAT }\end{array}$ & $\begin{array}{l}\text { Fampyra }{ }^{\circledR} \text { vs. Azetazo- } \\
\text { lamid vs. Placebo }\end{array}$ & Laufende Studie \\
\hline 7 & Zerebelläre Ataxien & ALCAT & $\begin{array}{l}\text { Acetyl-DL-Leucin } \\
\text { ( } 5 \mathrm{~g} / \mathrm{d} \text { vs. Placebo })\end{array}$ & $\begin{array}{l}\text { Patientenrekrutierung } \\
\text { ab ca. Oktober } 2015\end{array}$ \\
\hline 8 & $\begin{array}{l}\text { Zerebelläre Gang- } \\
\text { störungen }\end{array}$ & FACEG & Fampyra ${ }^{\circledR}$ vs. Placebo & Laufende Studie \\
\hline
\end{tabular}

Tab. 3 Derzeit am Deutschen Schwindelzentrum durchgeführte prospektive Placebo-kontrollierte Pharmakotherapiestudien bei verschiedenen Schwindelsyndromen. Es handelt sich jeweils um „investigator-initiated trials" (IITs), 1 - 7 gefördert vom Bundesministerium für Bildung und Forschung (BMBF). 
0,98\% [58,59]. Die diagnostischen Kriterien für die vestibuläre Migräne wurden definiert durch die Bárány-Gesellschaft zusammen mit der Internationalen Klassifikation von Kopfschmerzerkrankungen (ICHD) und sind wie folgt: A) mindestens 5 Episoden mit vestibulären Symptomen von mittelschwerer bis schwerer Intensität, Dauer: 5 min bis 72 Stunden; B) positive Anamnese einer Migräne mit oder ohne Aura nach den Diagnosekriterien der ICHD; C) mindestens ein migränetypisches Begleitsymptom bei mindestens 50\% der vestibulären Attacken: Kopfschmerzen mit mindestens zwei der folgenden Merkmale (einseitige Lokalisation, pulsierender Charakter, mäßige bis starke Schmerzintensität, Verstärkung durch körperliche Routineaktivitäten); Photophobie und Phonophobie; visuelle Aura; D) nicht besser durch eine andere vestibuläre Diagnose erklärt. Eine solche operationalisierte Klassifizierung ist wichtig für übereinstimmende Diagnosen und damit ebenfalls für zukünftige klinische Studien [60].

Bei der vestibulären Migräne gibt es bislang nur eine Studie zur Wirksamkeit von Flunarizin [61], zu weiteren Medikamenten gibt es bislang keine randomisierten kontrollierten Studien [62]. Die medikamentöse Behandlung bei der vestibulären Migräne wird aufgrund dessen in Analogie zur Migräne ohne Aura empfohlen. Darüber hinaus wird zurzeit eine prospektive randomisierte multizentrische Placebo-kontrollierte Studie (PROVEMIG) mit Metoprolol (95 mg täglich im Vergleich zu Placebo) durchgeführt ( $\odot$ Tab.3).

Episodische Ataxie Typ 2 und andere Formen familiärer episodischer Ataxien

Die episodische Ataxie Typ 2 (EA 2) ist eine seltene, autosomaldominante Ionenkanalerkrankung und der häufigste Subtyp von episodischen Ataxien. In etwa 60 \% der Patienten lassen sich Mutationen des CACNA1A-Gens nachweisen, das die alpha-Untereinheit des P/Q-Typ-Calciumkanals kodiert [63, 64].

Klinisch treten typischerweise rezidivierende Anfälle von Schwindel mit ataktischen Symptomen auf, die meist durch Stress, Anstrengung oder Alkohol provoziert werden und dauerhaft für viele Stunden anhalten. Ein weiteres wichtiges Merkmal sind Kleinhirnfunktionsstörungen im Intervall. Bei mehr als $90 \%$ der Patienten können zentrale Augenbewegungsstörungen dokumentiert werden wie eine sakkadierte Blickfolge, Blickhaltedefizite mit Blickrichtungsnystagmus sowie eine Beeinträchtigung der Fixationssuppression, vor allem auch ein Downbeat-Nystagmus [64]. Diese eindeutigen klinischen Zeichen erlauben es auch, EA2-Patienten von Patienten mit einer Migräne zu unterscheiden, die lediglich ganz diskrete zentrale Okulomotorikstörungen im Intervall haben können [65, 66].

In der Vergangenheit war die Therapie der Wahl die Einnahme des Carboanhydraseinhibitors Azetazolamid in einer Dosierung von 250 - $1000 \mathrm{mg}$ pro Tag [67]. Über Veränderungen des pH-Werts und damit des Transmembranpotenzials führt diese Medikation zu einer Veränderung der Erregbarkeit der Neurone. Bislang gibt es keine klinisch randomisierten Studien zur Wirksamkeit von Azetazolamid. Auf der anderen Seite ist das Medikament für zahlreiche dosisabhängige Nebenwirkungen bekannt (z. B. Nierensteine, Nephrokalzinose, vermehrtes Schwitzen, Parästhesien, Muskelversteifung mit leichter Ermüdbarkeit und gastrointestinale Störungen), die zu einer erheblich eingeschränkten Verwendung in der klinischen Praxis führen.

Die empfohlene Therapie der Wahl ist der reversible Kaliumkanalblocker 4-Aminopyridin (4-AP). Das Therapieprinzip besteht in der Aktivierung von Purkinjezellen durch die Blockade von Kaliumkanälen. Zudem verstärkt die Normalisierung der irregulä- ren spontanen Aktivität den inhibitorischen Einfluss von GABA auf vestibuläre und zerebelläre Kerngebiete [68]. Bereits im Jahr 2004 zeigte eine Beobachtungsstudie an drei Patienten eine Verringerung der Attackenfrequenz und -intensität [69]. Diese Ergebnisse konnten in einer randomisierten klinischen Studie bei Patienten mit EA2 und anderen familiären Ataxien bestätigt werden; gleichzeitig erhöhte sich durch die Behandlung die Lebensqualität, was bei einer symptomatischen Therapie von großer Bedeutung ist [70]. Die empfohlene Dosierung liegt bei 5 bis $10 \mathrm{mg}$ dreimal täglich (15 bis $30 \mathrm{mg}$ Tagesdosis). Die Retardform des 4-AP ist ebenfalls gut wirksam und verträglich im Rahmen einer Off-Label-Behandlung [71]. Zurzeit wird eine randomisierte klinische Studie bei EA2 durchgeführt (EAT-2-TREAT) ( $\bullet$ Tab.3), die die Wirksamkeit von Azetazolamid und retardiertem 4-Aminopyridin (Fampyra ${ }^{\circledR}$ ) gegenüber Placebo untersucht.

Downbeat-Nystagmus (DBN), Upbeat-Nystagmus (UBN) und andere zentrale Nystagmusformen

Sowohl der DBN als auch der UBN zeigen immer eine zentrale Störung an und haben eine lokalisatorische Bedeutung. Klinisch manifestieren sich die Erkrankungen primär als Schwankschwindel mit Gangunsicherheit; Oszillopsien und Sehstörungen treten meist erst im Verlauf auf. Im Gegensatz zum peripheren vestibulären Spontannystagmus handelt es sich bei beiden Formen um sogenannte Fixationsnystagmen; das heißt, die Intensität nimmt bei Fixation eher zu und nicht ab. Der Downbeat-Nystagmus ist der häufigste erworbene persistierende Nystagmus [72]. Ihm liegt meist eine beidseitige Flocculusfunktionsstörung zugrunde [73]. Die drei häufigsten Ursachen sind zerebelläre Atrophie, Ischämie und Arnold-Chiari-Malformation [72].

Aminopyridine: In einer randomisierten klinischen Studie 2003 wurde gezeigt, dass 3,4-Diaminopyridin, ein nicht-selektiver Kaliumkanal-Blocker, die Intensität des DBN reduziert. Diese Ergebnisse wurden im Verlauf bestätigt [74 - 76]. 4-Aminopyridine haben das gleiche Wirkprinzip, wobei 4-AP dem 3,4-DAP überlegen ist, da die Substanz mehr lipidlöslich ist und damit die Blut-HirnSchranke leichter überwindet [77].

Eine neuere randomisierte klinische Studie mit 4-AP bei Patienten mit DBN zeigte eine Verringerung der Geschwindigkeit der langsamen Phase des DBN um etwa $50 \%$ und damit eine Verbesserung der Sehschärfe (Dosierung 5 mg zwei- bis viermal täglich). Einschränkend ist zu sagen, dass eine subjektive Besserung nicht beschrieben wurde, vermutlich aufgrund der nur kurzen Halbwertszeit des Medikaments [78]. Die kurze Halbwertszeit wurde mit der retardierten Form (Fampyra ${ }^{\circledR}$ ) behoben.

Darauf basierend besteht die Empfehlung zur Behandlung des DBN mit 4-Aminopyridin (Dosierung $5 \mathrm{mg}$ zwei- bis viermal täglich); auch die retardierte Form, die für die Behandlung von Gangstörungen bei MS zugelassen ist, kann verwendet werden. Chlorzoxazone ist ein unspezifischer Aktivator Calcium-aktivierter Kaliumkanäle. Diese Substanz wird in den USA „over the counter“ zur Behandlung von Muskelkrämpfen eingesetzt, in Deutschland ist sie über internationale Apotheken ebenfalls zu beziehen, wobei bislang keine Erfahrungen zu Kostenübernahmen durch die Krankenkassen vorliegen In Tiermodellen der Episodischen Ataxie Typ 2 (sog. „Tottering mouse“) war ein positiver Effekt auf die Attackenfrequenz gefunden worden [68]. Auf der Basis dieser Daten wurde die Substanz bei Patienten mit zerebellärem DBN untersucht. Chlorzoxazone $(3 \times 500 \mathrm{mg}$ pro Tag) führte zu einer signifikanten Abnahme der Intensität des DBN und einer Verbesserung der Standregulation [79]. Dieses Medikament ist eine Alternative zum 4-Aminopyridin, insbesondere wenn 
bei Patienten Herzrhythmusstörungen oder andere Erkrankungen vorliegen, die eine Behandlung mit Aminopyridinen unmöglich machen.

Der Upbeat-Nystagmus (UBN) ist deutlich seltener als der DBN und wird durch paramediane medulläre oder pontomesenzephale Läsionen verursacht, wie z. B. bei Hirnstamminfarkten oder -blutungen. Im Gegensatz zum DBN hält der UBN meist nur einige Wochen an. Auch beim UBN ist 4-AP möglicherweise wirksam. Allerdings gibt es dazu nur eine Einzelfallbeschreibung [80]. Die Behandlung wurde vom Patienten gut vertragen. Darüber hinaus konnten in einer Anwendungsbeobachtung auch positive Effekte von Baclofen (15-30 mg/d) gefunden werden [81].

Auch zum zentralen Lagerungsschwindel gibt es einen Fallbericht zu positiven Effekten von 4-Aminopyridin; parallel dazu kam es zu einer Erhöhung der metabolischen Aktivität im Flocculus/Paraflocculus, wie mittel FDG-PET gezeigt werden konnte [82].

\section{Zerebelläre Ataxien}

Die Behandlung der motorischen Symptome von degenerativen Kleinhirnerkrankungen ist nach wie vor schwierig. Es besteht Einigkeit darüber, dass bisher keine wirksamen Medikamente nachgewiesen worden sind [57]. Physiotherapie führt zu einer klinischen Stabilisierung und wird deshalb auf regelmäßiger und intensiver Basis empfohlen [83].

4-Aminopyridine: Es wurden die Effekte von 4-Aminopyridin bei zerebellären Gangstörungen unterschiedlicher Ätiologie mittels differenzierter Ganganalyse untersucht. 4-Aminopyridin führte zu einer signifikanten Abnahme der Gangvariabilität und damit der Sturzgefahr [84]. Diese Befunde werden derzeit im Rahmen einer Placebo-kontrollierten Studie (FACEG) näher untersucht.

Acetyl-DL-Leucin: Seit 1957 wird die modifizierte Aminosäure Acetyl-DL-Leucin in Frankreich zur symptomatischen Behandlung von Schwindel eingesetzt. In Frankreich ist sie frei erhältlich, in Deutschland über internationale Apotheken zu bestellen, bei einzelnen Patienten mit zerebellären Erkrankungen haben die Krankenkassen die Kosten der Behandlung im Rahmen eines Off-Label-Gebrauchs übernommen. Basierend auf Einzelbeobachtungen, wurde dieses Präparats in einer Dosierung von $5 \mathrm{~g}$ pro Tag bei 13 Patienten mit zerebellärer Ataxie unterschiedlicher Ätiologie gegeben. Nach einer Woche fand sich eine signifikante und klinisch relevante Verbesserung in allen eingesetzten Ataxie-Scores (sog. SARA und SCAFI); parallel dazu verbesserte sich die Lebensqualität [85]. In der weiteren Fallserie mit Patienten mit Niemann-Pick Typ C zeigte sich ebenfalls eine klinische Besserung [86], wohingegen in einer Fallserie mit 10 Patienten mit einer zerebellären Ataxie unterschiedlicher Ätiologie zwar bei 7 Patienten eine subjektive Verbesserung beschrieben wurde, jedoch keine objektivierbare gemessen in den Ataxie-Scores objektivierbare. Der Unterschied dieser Fallserie war jedoch die nur einmal täglich erfolgte Einnahme der flüssigen Form von Acetyl-DL-Leucin [87]. Es wird postuliert, dass diese Substanz offensichtlich durch Einlagerung in die Zellmembran und Interaktion mit Phospholipiden zu einer Normalisierung des Membranpotenzials führt [25]. Die Wirksamkeit wird gerade in einer Placebo-kontrollierten multizentrischen Studie untersucht (ALCAT) ( Tab. 3).

\section{Fazit für die Praxis}

\section{$\nabla$}

Schwindel ist das zweithäufigste Leitsymptom in der Praxis. Nach ihrer Ursache werden zentrale vestibuläre von peripheren vestibulären Schwindelsyndromen unterschieden. Eine präzise Diagnose ist die Voraussetzung für eine wirksame medikamentöse Therapie von Schwindel und Nystagmus. Antivertiginosa und Antiemetika sollen nur zur symptomatischen Kurzzeitbehandlung eingesetzt werden. In klinischen Studien der letzten zehn Jahre wurden für folgende Erkrankungen medikamentöse Therapieansätze entwickelt, wobei noch vielfach eine verlässliche Evidenz durch randomisierte klinische Studien fehlt: Glukokortikoide für die akute Neuritis vestibularis, Betahistin in hoher Dosierung und als Langzeittherapie für die Prophylaxe des Morbus Menière, Carbamazepin und Oxcarbazepin in niedriger Dosierung bei der Vestibularisparoxysmie, Aminopyridine bei Downbeat-Nystagmus, Upbeat-Nystagmus und Episodischer Ataxie Typ 2.

\section{Take Home Message}

Viele Formen von Schwindel und Nystagmus können heute therapiert werden, jedoch fehlen noch randomisierte Placebo-kontrollierte klinische Studien z. B. zur symptomatischen Behandlung akuten Schwindels, vestibulärer Migräne, Vestibularisparoxysmie oder funktionellen Schwindels. Grundvoraussetzung für eine wirksame medikamentöse Therapie der verschiedenen Schwindelsyndrome und Nystagmusformen ist zunächst eine genaue Diagnose, da für die jeweiligen zugrunde liegenden Erkrankungen verschiedene spezifische Medikamente zur Verfügung stehen.

Interessenkonflikte: M. Strupp ist Herausgeber folgender Zeitschriften: Journal of Neurology, Frontiers of Neuro-otology und F1000. Er bezieht Sprechergelder von Abbott, Actelion, UCB, GSK, TEVA, Biogen, Pierre-Fabre, Heel, Eisai, MSD und Hennig Pharma. K. Feil, N. Böttcher, O. Kremmyda, C. Muth und Julian Teufel geben an, dass kein Interessenkonflikt besteht.

Zahlreiche der beschriebenen Studien werden finanziert durch das BMBF (Grant No.01E00901 and 01E01401).

\section{Literatur}

1 Strupp M, Thurtell MJ, Shaikh AG et al. Pharmacotherapy of vestibular and ocular motor disorders, including nystagmus. J Neurol 2011; 258: $1207-1222$

2 Strupp M, Kremmyda O, Brandt T. Pharmacotherapy of vestibular disorders and nystagmus. Semin Neurol 2013; 33: 286-296

3 Brandt T, Dieterich M, Strupp M. Vertigo - Leitsymptom Schwindel. 2. Auflage. Heidelberg: Springer-Verlag; 2012

4 Beck R, Gunther L, Xiong $G$ et al. The mixed blessing of treating symptoms in acute vestibular failure-evidence from a 4-aminopyridine experiment. Exp Neurol 2014; 261: 638-645

5 Shaia WT, Diaz RC. Evolution in surgical management of superior canal dehiscence syndrome. Curr Opin Otolaryngol Head Neck Surg 2013; 21: 497-502

6 McDonnell MN, Hillier SL. Vestibular rehabilitation for unilateral peripheral vestibular dysfunction. Cochrane Database Syst Rev 2015, CD005397 1

7 Baloh RW. Clinical practice. Vestibular neuritis. N Eng J Med 2003; 348: $1027-1032$

8 Baloh RW, Ishyama A, Wackym PA et al. Vestibular neuritis: clinicalpathologic correlation. Otolaryngol Head Neck Surg 1996; 114: 586 592

9 Schuknecht HF, Gacek MR. The three faces of vestibular ganglionitis. Ann Otol Rhinol Laryngol 2002; 111: $103-114$

10 Gacek RR, Schuknecht HF. Pathology of the ear. 2 ed. Phildelphia: Lea \& Febinger; 1993

11 Nadol JB Jr. Vestibular neuritis. Otolaryngol Head Neck Surg 1995; 112: $162-172$ 
12 Schuknecht HF, Kitamura K. Second Louis H. Clerf Lecture. Vestibular neuritis. The Annals of otology, rhinology \& laryngology. Supplement 1981; 90: 1-19

13 Arbusow V, Derfuss T, Held K et al. Latency of herpes simplex virus type-1 in human geniculate and vestibular ganglia is associated with infiltration of CD8+ T cells. J Med Virol 2010; 82: 1917-1920

14 Arbusow V, Schulz P, Strupp M et al. Distribution of herpes simplex virus type 1 in human geniculate and vestibular ganglia: implications for vestibular neuritis. Ann Neurol 1999; 46: 416-419

15 Arbusow V, Theil D, Strupp M et al. HSV-1 not only in human vestibular ganglia but also in the vestibular labyrinth. Audiol Neurootol 2001; 6: $259-262$

16 Theil D, Arbusow V, Derfuss T et al. Prevalence of HSV-1 LAT in human trigeminal, geniculate, and vestibular ganglia and its implication for cranial nerve syndromes. Brain Pathol 2001; 11: 408-413

17 Bisdorff A, Von Brevern M, Lempert T et al. Classification of vestibular symptoms: towards an international classification of vestibular disorders. J Vestib Res 2009; 19: 1-13

18 Strupp M, Zingler VC, Arbusow V et al. Methylprednisolone, valacyclovir, or the combination for vestibular neuritis. N Engl J Med 2004; 351: $354-361$

19 Goudakos JK, Markou KD, Franco-Vidal V et al. Corticosteroids in the treatment of vestibular neuritis: a systematic review and meta-analysis. Otol Neurotol 2010; 31: 183-189

20 Karlberg ML, Magnusson M. Treatment of acute vestibular neuronitis with glucocorticoids. Otol Neurotol 2011; 32: 1140-1143

21 Goudakos JK, Markou KD, Psillas G et al. Corticosteroids and vestibular exercises in vestibular neuritis. Single-blind randomized clinical trial. JAMA Otolaryngol Head Neck Surg 2014; 140: 434-440

22 Fishman JM, Burgess C, Waddell A. Corticosteroids for the treatment of idiopathic acute vestibular dysfunction (vestibular neuritis). Cochrane Database Syst Rev 2011; DOI: 10.1002/14651858.CD008607 pub2: CD008607

23 Wegner I, van Benthem PP, Aarts MC et al. Insufficient evidence for the effect of corticosteroid treatment on recovery of vestibular neuritis. Otolaryngol Head Neck Surg 2012; 147: 826-831

24 Dutia MB. Mechanisms of vestibular compensation: recent advances. Curr Opin Otolaryngol Head Neck Surg 2010; 18: 420-424

25 Vibert $N$, Vidal PP. In vitro effects of acetyl-DL-leucine (tanganil) on central vestibular neurons and vestibulo-ocular networks of the guinea-pig. Eur J Neurosci 2001; 13: 735-748

26 Strupp M, Arbusow V, Maag KP et al. Vestibular exercises improve central vestibulospinal compensation after vestibular neuritis. Neurology 1998; $51: 838-844$

27 Herdman SJ. Vestibular rehabilitation. Curr Opin Neurol 2013; 26: 96 101

28 Minor LB, Schessel DA, Carey JP. Meniere's disease. Curr Opin Neurol 2004; $17: 9-16$

29 Semaan MT, Alagramam KN, Megerian CA. The basic science of Meniere's disease and endolymphatic hydrops. Curr Opin Otolaryngol Head Neck Surg 2005; 13: $301-307$

30 Sajjadi H, Paparella MM. Meniere's disease. Lancet 2008; 372: 406 414

31 Merchant SN, Adams JC, Nadol JB et al. Pathophysiology of Meniere's syndrome: are symptoms caused by endolymphatic hydrops? Otol Neurotol 2005; 26: 74-81

32 Yeh TH, Herman P, Tsai MC et al. A cationic nonselective stretch-activated channel in the Reissner's membrane of the guinea pig cochlea. Am J Physiol 1998; 274: C566-C576

33 Thirlwall AS, Kundu S. Diuretics for Meniere's disease or syndrome. Cochrane Database Syst Rev 2006; DOI: 10.1002/14651858.CD003599. pub2: CD003599

34 Pullens B, Verschuur HP, van Benthem PP. Surgery for Meniere's disease. Cochrane Database Syst Rev 2013, CD005395 2

35 Arrang JM, Garbarg M, Quach TT et al. Actions of betahistine at histamine receptors in the brain. Eur J Pharmacol 1985; 111: $73-84$

36 Gbahou F, Davenas E, Morisset $S$ et al. Effects of betahistine at histamine $\mathrm{H} 3$ receptors: mixed inverse agonism/agonism in vitro and partial inverse agonism in vivo. J Pharmacol Exp Thers 2010; 334: 945-954

37 Dziadziola JK, Laurikainen EL, Rachel JD et al. Betahistine increases vestibular blood flow. Otolaryngol Head Neck Surg 1999; 120: 400 - 405

38 Bertlich M, Ihler F, Sharaf K et al. Betahistine metabolites, Aminoethylpyridine, and Hydroxyethylpyridine increase cochlear blood flow in guinea pigs in vivo. Int J Audiol 2014; 53: 753 -759
39 Strupp $M$, Hupert D, Frenzel C et al. Long-term prophylactic treatment of attacks of vertigo in Meniere's disease-comparison of a high with a low dosage of betahistine in an open trial. Acta Otolaryngol 2008; 128 : $520-524$

40 Lezius F, Adrion C, Mansmann U et al. High-dosage betahistine dihydrochloride between 288 and $480 \mathrm{mg} /$ day in patients with severe Meniere's disease: a case series. Eur Arch Otorhinolaryngol 2011; 268 : $1237-1240$

41 James AL, Burton MJ. Betahistine for Meniere's disease or syndrome. Cochrane Database Syst Rev 2001; DOI: 10.1002/14651858.CD001873: CD001873

42 Nauta JJ. Meta-analysis of clinical studies with betahistine in Meniere's disease and vestibular vertigo. Eur Arch Otorhinolaryngol 2014; 271: $887-897$

43 Postema RJ, Kingma CM, Wit HP et al. Intratympanic gentamicin therapy for control of vertigo in unilateral Meniere's disease: a prospective, double-blind, randomized, placebo-controlled trial. Acta Otolaryngol 2008; $128: 876-880$

44 Stokroos R, Kingma H. Selective vestibular ablation by intratympanic gentamicin in patients with unilateral active Meniere's disease: a prospective, double-blind, placebo-controlled, randomized clinical trial. Acta Otolaryngol 2004; 124: $172-175$

45 Diamond C, O'Connell DA, Hornig JD et al. Systematic review of intratympanic gentamicin in Meniere's disease. J Otolaryngol 2003; 32: $351-361$

46 Huon $L K$, Fang $T Y$, Wang PC. Outcomes of intratympanic gentamicin injection to treat Meniere's disease. Otol Neurotol 2012; 33: 706 - 714

47 Colletti V, Carner M, Colletti L. Auditory results after vestibular nerve section and intratympanic gentamicin for Meniere's disease. Otol Neurotol 2007; 28: $145-151$

48 Huppert D, Strupp M, Brandt T. Long-term course of Meniere's disease revisited. Acta Otolaryngol 2010; 130: 644-651

49 Phillips JS, Westerberg B. Intratympanic steroids for Meniere's disease or syndrome. Cochrane Database Syst Rev 2011; DOI: 10.1002/14651858. CD008514.pub2: CD008514

50 Garduno-Anaya MA, Couthino De Toledo H, Hinojosa-Gonzalez R et al. Dexamethasone inner ear perfusion by intratympanic injection in unilateral Meniere's disease: a two-year prospective, placebo-controlled double-blind, randomized trial. Otolaryngol Head Neck Surg 2005; 133: $285-294$

51 Gabra N, Saliba I. The effect of intratympanic methylprednisolone and gentamicin injection on Meniere's disease. Otolaryngol Head Neck Surg 2013; 148: 642-647

52 Brandt T, Dieterich $M$. Vestibular paroxysmia: vascular compression of the eighth nerve? Lancet 1994; 343: 798 - 799

53 Hufner K, Barresi D, Glaser M et al. Vestibular paroxysmia: diagnostic features and medical treatment. Neurology 2008; 71: 1006-1014

54 Moller MB, Moller AR, Jannetta PJ et al. Diagnosis and surgical treatment of disabling positional vertigo. Journal of neurosurgery 1986; 64: $21-28$

55 Strupp M, von Stuckrad-Barre S, Brandt T et al. Teaching neuroimages: Compression of the eighth cranial nerve causes vestibular paroxysmia. Neurology 2013; 80: e77

56 Brandt T, Dieterich $M$. VIIIth nerve vascular compression syndrome: vestibular paroxysmia. Bailliere's clinical neurology 1994; 3: 565-575

57 Ilg W, Bastian AJ, Boesch S et al. Consensus paper: management of degenerative cerebellar disorders. Cerebellum 2014; 13: 248-268

58 Neuhauser HK. Epidemiology of vertigo. Curr Opin Neurol 2007; 20: 40-46

59 Neuhauser HK. Epidemiology of dizziness and vertigo. Nervenarzt 2009; 80: 887-894

60 Lempert T, Olesen J, Furman J et al. Vestibular migraine: diagnostic criteria. Consensus document of the Barany Society and the International headache society. Rev Neurol (Paris) 2014; 170: 401 -406

61 Lepcha A, Amalanathan S, Augustine AM et al. Flunarizine in the prophylaxis of migrainous vertigo: a randomized controlled trial. Eur Arch Otorhinolaryngol 2014; 271: 2931 - 2936

62 Maldonado Fernandez M, Birdi JS, Irving GJ et al. Pharmacological agents for the prevention of vestibular migraine. Cochrane Database Syst Rev 2015, CD010600 6

63 Ophoff RA, Terwindt GM, Vergouwe MN et al. Familial hemiplegic migraine and episodic ataxia type- 2 are caused by mutations in the $\mathrm{Ca} 2+$ channel gene CACNL1A4. Cell 1996; 87: 543-552

64 Jen JC. Hereditary episodic ataxias. Ann N Y Acad Sci 2008; 1142: 250 253 
65 Strupp M, Zwergal A, Brandt T. Episodic ataxia type 2. Neurotherapeutics 2007; 4: 267-273

66 Jen JC, Baloh RW. Familial episodic ataxia: a model for migrainous vertigo. Ann N Y Acad Sci 2009; 1164: 252-256

67 Jen J, Kim GW, Baloh RW. Clinical spectrum of episodic ataxia type 2 . Neurology 2004; 62: 17-22

68 Alvina $K$, Khodakhah $K$. The therapeutic mode of action of 4-aminopyridine in cerebellar ataxia. J Neurosci 2010; 30: 7258-7268

69 Strupp M, Kalla R, Dichgans $M$ et al. Treatment of episodic ataxia type 2 with the potassium channel blocker 4-aminopyridine. Neurology 2004; 62: $1623-1625$

70 Strupp M, Kalla R, Claassen J et al. A randomized trial of 4-aminopyridine in EA2 and related familial episodic ataxias. Neurology 2011; 77: 269-275

71 Claassen J, Teufel J, Kalla $R$ et al. Effects of dalfampridine on attacks in patients with episodic ataxia type 2 : an observational study. J Neurol 2013; 260: 668-669

72 Wagner JN, Glaser M, Brandt T et al. Downbeat nystagmus: aetiology and comorbidity in 117 patients. J Neurol Neurosurg Psychiatry 2008; 79: $672-677$

73 Kalla R, Deutschlander A, Hufner K et al. Detection of floccular hypometabolism in downbeat nystagmus by fMRI. Neurology 2006; 66: 281 283

74 Sprenger A, Zils E, Rambold $\mathrm{H}$ et al. Effect of 3,4-diaminopyridine on the postural control in patients with downbeat nystagmus. Ann N Y Acad Sci 2005; 1039: 395-403

75 trupp M, Schuler O, Krafczyk S et al. Treatment of downbeat nystagmus with 3,4-diaminopyridine: a placebo-controlled study. Neurology 2003; 61: $165-170$

76 Helmchen C, Gottschalk S, Sander T et al. Beneficial effects of 3,4-diaminopyridine on positioning downbeat nystagmus in a circumscribed uvulo-nodular lesion. J Neurol 2007; 254: 1126-1128
77 Kalla R, Spiegel R, Claassen J et al. Comparison of 10-mg doses of 4-aminopyridine and 3,4-diaminopyridine for the treatment of downbeat nystagmus. J Neuroophthalmol 2011; 31: 320 - 325

78 Claassen J, Spiegel R, Kalla $R$ et al. A randomised double-blind, crossover trial of 4-aminopyridine for downbeat nystagmus-effects on slowphase eye velocity, postural stability, locomotion and symptoms. J Neurol Neurosurg Psychiatry 2013; 84: 1392 -1399

79 Feil KCJ, Bardins S, Teufel J et al. Effect of chlorzoxazone in patients with downbeat nystagmus: a pilot-trial. Neurology 2013; 81: 1152 -1158

80 Glasauer S, Kalla $R$, Buttner $U$ et al. 4-aminopyridine restores visual ocular motor function in upbeat nystagmus. J Neurol Neurosurg Psychiatry 2005; 76: $451-453$

81 Dieterich $M$, Straube A, Brandt T et al. The effects of baclofen and cholinergic drugs on upbeat and downbeat nystagmus. J Neurol Neurosurg Psychiatry 1991; 54: 627-632

82 Kremmyda O, Zwergal A, la Fougere C et al. 4-Aminopyridine suppresses positional nystagmus caused by cerebellar vermis lesion. J Neurol 2013; 260: $321-323$

83 Ilg W, Synofzik M, Brotz D et al. Intensive coordinative training improves motor performance in degenerative cerebellar disease. Neurology 2009; 73: $1823-1830$

84 Schniepp R, Wuehr M, Ackl N et al. 4-Aminopyridine improves gait variability in cerebellar ataxia due to CACNA 1A mutation. J Neurol 2011; 258: $1708-1711$

85 Strupp M, Teufel J, Habs $M$ et al. Effects of acetyl-DL-leucine in patients with cerebellar ataxia: a case series. J Neurol 2013; 260: 2556-2561

86 Bremova TMV, Amraoui Y, Mengel E et al. Acetyl-DL-leucine in Niemann-Pick type C: a case series. Neurology 2015, In press

87 Pelz JO, Fricke C, Saur D et al. Failure to confirm benefit of acetyl-DLleucine in degenerative cerebellar ataxia: a case series. J Neurol 2015; 262: $1373-1375$ 